University of Nebraska - Lincoln

DigitalCommons@University of Nebraska - Lincoln

USDA Forest Service / UNL Faculty Publications U.S. Department of Agriculture: Forest Service -National Agroforestry Center

2012

\title{
Tree and impervious cover in the United States
}

David J. Nowak

USDA Forest Service, dnowak@fs.fed.us

Eric J. Greenfield

USDA Forest Service, ejgreenfield@fs.fed.us

Follow this and additional works at: https://digitalcommons.unl.edu/usdafsfacpub

Nowak, David J. and Greenfield, Eric J., "Tree and impervious cover in the United States" (2012). USDA Forest Service / UNL Faculty Publications. 239.

https://digitalcommons.unl.edu/usdafsfacpub/239

This Article is brought to you for free and open access by the U.S. Department of Agriculture: Forest Service -National Agroforestry Center at DigitalCommons@University of Nebraska - Lincoln. It has been accepted for inclusion in USDA Forest Service / UNL Faculty Publications by an authorized administrator of DigitalCommons@University of Nebraska - Lincoln. 


\title{
Tree and impervious cover in the United States
}

\author{
David J. Nowak*, Eric J. Greenfield \\ USDA Forest Service, Northern Research Station, 5 Moon Library, SUNY-ESF, Syracuse, NY 13210, United States
}

\section{A R T I C L E I N F O}

\section{Article history:}

Received 29 June 2011

Received in revised form 6 April 2012

Accepted 10 April 2012

Available online 9 May 2012

\section{Keywords:}

Urban tree cover

Rural tree cover

State tree cover

Urban impervious cover

Rural impervious cover

State impervious cover

\begin{abstract}
A B S T R A C T
Using aerial photograph interpretation of circa 2005 imagery, percent tree canopy and impervious surface cover in the conterminous United States are estimated at $34.2 \%$ (standard error $(\mathrm{SE})=0.2 \%$ ) and $2.4 \%$ $(\mathrm{SE}=0.1 \%)$, respectively. Within urban/community areas, percent tree cover $(35.1 \%, \mathrm{SE}=0.4 \%)$ is $\mathrm{simi}-$ lar to the national value, but percent impervious cover is significantly higher $(17.5 \%, \mathrm{SE}=0.3 \%)$. Tree cover per capita in urban areas averaged $377 \mathrm{~m}^{2} /$ person, while impervious cover per capita averaged $274 \mathrm{~m}^{2} /$ person. Percent tree cover in urban/community areas tends to be significantly higher than in rural areas in several predominantly grassland states, with the greatest difference in Kansas (+17.3\%). Most states in more forested regions exhibited a decrease in tree cover between urban/community areas and rural lands, with greatest difference in Kentucky (-37.9\%). These changes in tree cover varied significantly among states, illustrating the roles of urban development patterns, management/planning interactions, and the natural environment on creating cover patterns exhibited in urban areas. Understanding these forces and patterns can lead to better planning and management activities to optimize the mix of tree and impervious cover to sustain urban functions while enhancing environmental quality and human health in urban areas.
\end{abstract}

Published by Elsevier B.V.

\section{Introduction}

Tree canopy and impervious surface cover affect ecosystem services provided by a landscape. These cover elements play particularly important roles in cities and towns in terms of their impacts on the physical and socio-economic environment, and, consequently, human health and well-being in these areas. Trees not only provide numerous economic and ecosystem services and values to a community, but also incur various economic or environmental costs. Trees supply ecosystem services associated with air and water quality, building energy conservation, moderation of air temperatures, reductions in ultraviolet radiation, and many other environmental and social benefits (e.g., Dwyer, McPherson, Schroeder, \& Rowntree, 1992; Kuo \& Sullivan, 2001; Nowak \& Dwyer, 2007; Westphal, 2003; Wolf, 2003). Costs associated with trees can be both economic (e.g., planting and maintenance, increased building energy costs) and environmental (e.g., pollen, volatile organic compound emissions) (Nowak \& Dwyer, 2007).

Likewise, impervious cover plays an important role in the landscape, particularly in developed areas. These surfaces, such as roads, buildings, sidewalks, and parking lots, facilitate transportation and provide shelter, but also can negatively impact the environment. Increased impervious surface area can enhance local tempera-

\footnotetext{
* Corresponding author. Tel.: +1 315448 3212; fax: +1 3154883216. E-mail addresses: dnowak@fs.fed.us (D.J. Nowak), ejgreenfield@fs.fed.us (E.J. Greenfield).
}

tures and heat islands effects (Heisler \& Brazel, 2010; Oke, 1989), which consequently affects building energy use, human comfort and health, ozone production, and pollutant emissions.

Impervious surfaces impede water infiltration rates (Hamilton \& Waddington, 1999; Pitt \& Lantri, 2000), and reduce percolation, water table levels, and stream baseflow regimes (Faulkner, Edmonds-Brown, \& Green, 2000; Lerner, 2002). Removal of forest cover and/or increased impervious area due to urbanization is known to increase stream flow and peak runoff in streams (Leopold, 1968; National Research Council, 2008). These changes in stream flows can lead to flooding, soil erosion, and sedimentation in streams (Anderson, 1970; McMahon \& Cuffney, 2000; Paul \& Meyer, 2001; Rose \& Peters, 2001; Urbonas \& Benik, 1995). As the volume of urban stormwater runoff increased throughout the United States from the increase in impervious surfaces, the quality of surface runoff has degraded substantially (U.S. EPA, 1983). Poorer water quality and increased temperatures due to impervious surfaces can significantly impact human health.

Quantifying tree and impervious cover within the United States is important for understanding the magnitude, distribution and variation of these landscape attributes nationally. By quantifying these cover attributes, better estimates of the impacts of these landscape cover elements can be ascertained, and improved landscape planning and management can be initiated. Understanding how cover types vary among states can also support the development of optimal cover recommendations to sustain natural ecosystem functions in urban areas. 
Various estimates of tree and impervious cover in the United States have been made in the past, often based on satellite data. The most recent estimates of tree and impervious cover estimates are based on the 2001 National Land Cover Database (NLCD) that provides free, $30 \mathrm{~m}$ resolution, percent tree canopy and percent impervious cover values for the conterminous United States derived from circa 2001 Landsat 7 imagery (MRLC, 2010). However, NLCD estimates of tree cover tend to underestimate tree cover, relative to photo-interpretation estimates, in the conterminous United States by an average of $9.7 \%(\mathrm{SE}=1.0 \%)$ and impervious cover by $1.4 \%(\mathrm{SE}=0.4 \%)$ with underestimates varying by region (Nowak \& Greenfield, 2010).

Google Earth ${ }^{\circledR}$ imagery provides a good means to assess overall cover as it offers nearly complete coverage of the conterminous United States with interpretable aerial images. The purpose of this paper is to determine the magnitude and variation in tree and impervious surface cover among states using aerial photointerpretation, and to quantify how these cover types vary overall and within and among rural and urban/community defined areas.

\section{Methods}

To determine the percent tree and impervious cover in the United States, photo-interpretation of Google Earth ${ }^{\circledR}$ imagery was conducted. This interpretation was done in various stages based on the area being analyzed: (a) a sampling of the urban/community area in the conterminous United States was conducted, (b) the conterminous United States was interpreted within 65 NLCD mapping zones (Nowak \& Greenfield, 2010) to determine cover in rural areas, and (c) an analysis of rural and urban/community areas in Alaska and Hawaii was conducted.

\subsection{Photo-interpretation}

Within each area of analysis, random points were laid and interpreted to classify the cover type of each point on the Google Earth $^{\circledR}$ image. Trained photo interpreters with experience interpreting leaf-off and leaf-on imagery classified each point as trees/large shrubs (yes/no), impervious surface (yes/no), or as a non-interpretable image. As tree and impervious cover designations are not mutually exclusive (e.g., tree cover over sidewalk or road), the photo interpreters were instructed to determine if the tree canopy covered an impervious surface, in which case it was classified as both tree and impervious. Most points (99.6\%), exclusive of Alaska and Hawaii, fell on images that were readily interpretable with a resolution of approximately $1 \mathrm{~m}$ or less. Points falling on imagery with medium to coarse resolution (e.g., $30 \mathrm{~m}$ resolution) or with atmospheric obstructions (clouds) were considered non-interpretable and not included in the final analysis. This relatively minor omission of points should not lead to any bias (excluding Alaska) as the non-interpretable points were scattered throughout the United States. Dates of Google Earth ${ }^{\circledR}$ imagery were circa 2005 and had varying dates.

Within each analyzed area, the percentage of tree or impervious cover $(p)$ was calculated as the number of sample points $(x)$ hitting the cover attribute divided by the total number of interpretable sample points $(n)$ within the area of analysis $(p=x / n)$. The standard error of the estimate (SE) was calculated as SE $=\sqrt{p(1-p) / n}$ (Lindgren \& McElrath, 1969). This method has been used to assess tree cover in many cities (e.g., Nowak et al., 1996).

Six photo-interpreters were used for these analyses. Photo interpretation results were verified by having a random $10 \%$ sample of the points reinterpreted by another photo-interpreter. Generally, the quality control checks resulted in high agreement with the mean percent of agreement of about $95 \%$. Some of the disagreements in audit results were due to image changes in Google imagery between the original interpretation and the audit.

\subsection{Photo-interpretation of urban/community areas}

Photo-interpretation was conducted to estimate tree and impervious cover only within urban/community areas of the conterminous United States. The definition of community is based on jurisdictional or political boundaries delimited by U.S. Census Bureau definitions of incorporated or designated places (U.S. Census Bureau, 2007). Community lands are places of established human settlement that may include all, some, or no urban land within their boundaries. The definition of urban is based on population density as delimited using the U.S. Census Bureau's (2007) definition: all territory, population, and housing units located within urbanized areas or urban clusters. Urbanized area and urban cluster boundaries encompass densely settled territories, which are described by one of the following:

- one or more block groups or census blocks with a population density of at least 386.1 people $/ \mathrm{km}^{2}$ (1000 people $/ \mathrm{mi}^{2}$ ),

- surrounding census blocks with a minimum population density of 193.1 people $/ \mathrm{km}^{2}$ (500 people $/ \mathrm{mi}^{2}$ ), or

- less densely settled blocks that form enclaves or indentations, or are used to connect discontinuous areas.

As urban land reveals the more heavily populated areas (population density-based definition) and community land has varying amounts of urban land that are recognized by their geopolitical boundaries (political definition), both definitions provide information related to human settlements and the forest resources within those settlements. As some urban land exists beyond community boundaries and not all community land is urban (i.e., communities are often a mix of urban and rural land), the category of "urban/community" was created to classify the union of these two geographically overlapping definitions where most people live (Fig. 1).

Within urban/community areas in the conterminous United States, 15,000 random points were laid for photo-interpretation of tree and impervious cover. Based on GIS boundaries, each point was also classified as whether the point was within urban land, community land, or both. If the sample size in urban land or community land within each state was less than 100 points, additional points were randomly sampled to reach a minimum sample size of 100 to attain a maximum potential standard error of $5 \%$ for these areas.

Estimates of tree and impervious cover in each state were then made for: (1) urban land, (2) community land and (3) urban/community land. In total, 15,299 points were sampled in urban/community land, with 2488 total points in urban land alone, 5856 points in community land alone, and 6955 in areas where urban and community land overlapped. Tree and impervious cover in urban areas were also divided by 2000 U.S. Census urban population to estimate the tree and impervious cover per capita in urban areas in each state. Spearman correlation was used to test for relationships between urban impervious cover and urban tree cover, between urban impervious cover and urban population density, and between urban tree cover and urban population density (alpha $=0.05$ ).

\subsection{Comparison of urban/community cover with rural cover by state}

Photo-interpretation of the lower 48 states was conducted as part of a project to test how well the 2001 National Land Cover Database (NLCD) tree and impervious cover maps estimated tree 


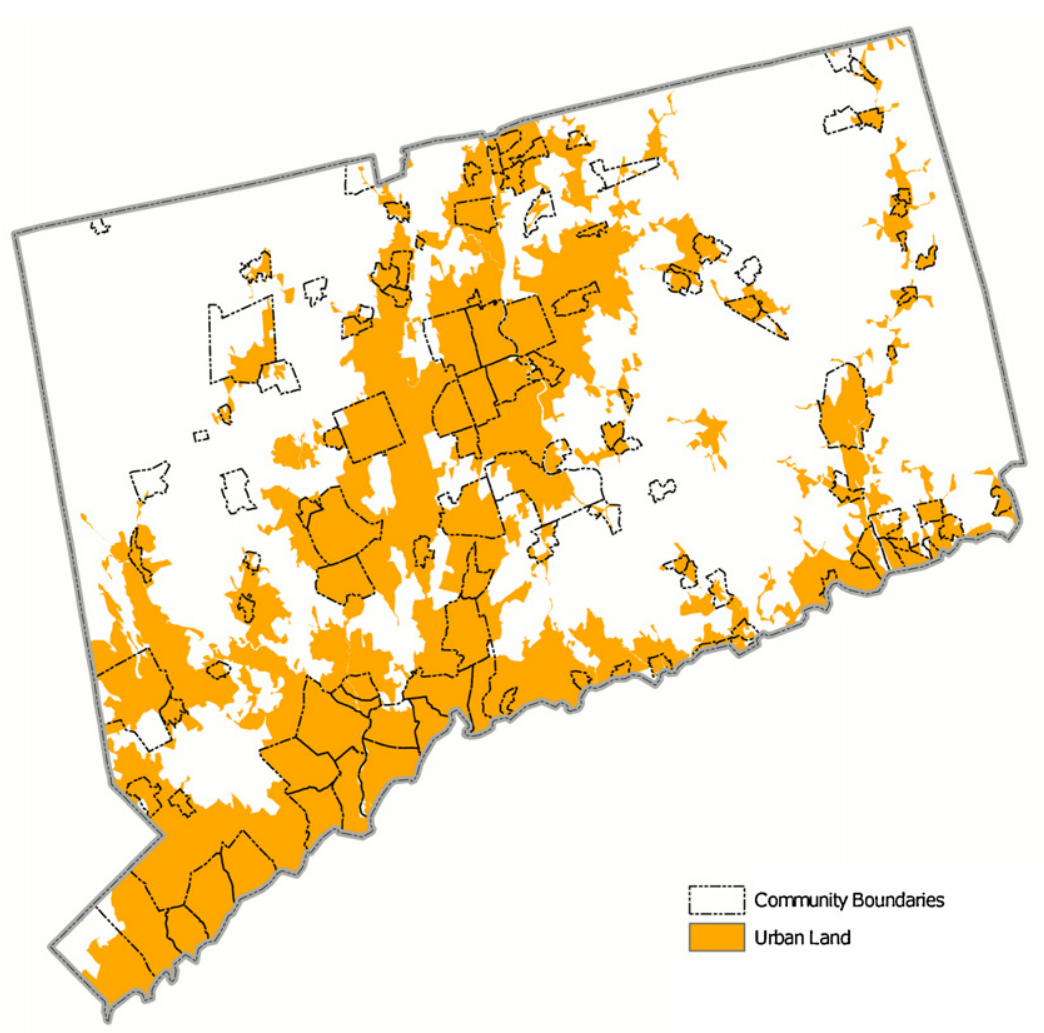

Fig. 1. Urban and community land in Connecticut (2000) (U.S. Census Bureau, 2007).

and impervious cover within 65 mapping zones that cover the conterminous United States (Nowak \& Greenfield, 2010). Within each mapping zone, 1000 randomly located points were converted and transformed into a Google Earth ${ }^{\circledR}$ compatible format (Google Inc., 2011) for photo-interpretation.

After photo-interpretation of the zones was completed, state boundaries were overlaid on the points to classify each point to its associated state. Because each mapping zone has a different density of points and because parts of different mapping zones could be within a state, each point was classified as to its state/mapping zone combination (e.g., NY, zone 63) and the area of each state/mapping zone combination was calculated using Geographic Information Systems (GIS). For each state/mapping zone combination that did not have a minimum of 50 sample points, additional points were randomly added and interpreted to reach the minimum sample size of 50 points. Additional points were only added to 66 small state/mapping zone polygons to attain the minimum sample size. In total, 66,986 points were interpreted for the conterminous United States. These points were used to determine cover in rural (i.e., non-urban/community areas) areas $(n=62,803)$ by weighting each state/mapping zone cover estimate and standard error by its zone land area within rural land to estimate the total rural percent cover (tree and impervious) and associated standard error.

To determine tree and impervious cover for each state, rural ( $n=62,803)$ and urban/community $(n=15,299)$ cover estimates and standard errors were weighted by its land area to estimate the total state percent cover (tree and impervious) and associated standard error (total $n=78,102$ ).

Percent tree or impervious cover and its 95\% confidence interval were calculated for rural land and urban/community land in each state. If the confidence intervals between rural and urban/community estimates did not overlap, then the difference between rural and urban/community tree or impervious cover was considered to be statistically significant. Spearman correlation was used to test the relationship between tree cover within urban areas and urban/community areas with overall state tree cover (alpha $=0.05)$.

\subsection{Photo-interpretation of Alaska and Hawaii}

As Alaska and Hawaii were not part of the original analyses of the conterminous United States, these states were analyzed separately. To analyze these states, 1000 random points were analyzed within urban land and a different 1000 points were analyzed in community areas. After this analysis, each point was classified as to whether it was within urban boundaries, community boundaries, and urban/community boundaries based on GIS census boundaries of these classes. Area of each class was also calculated using the GIS boundary files. Percent cover and standard error (SE) were calculated for each class and then weighted by the class area to produce a total estimate of percent cover and SE for each class type (i.e., urban, community, and urban/community).

For rural land, an additional 1000 points were interpreted. Due to poor image quality, the analysis of Alaska rural area and thus state total cover is not reported, as only $23.5 \%$ of the rural points could be interpreted. Also for Alaska, only $53.6 \%$ of the points in community land were interpretable, but these points were included in the analysis of community and urban/community cover. In Hawaii, $97.6 \%$ of the points were interpretable in urban/community areas and $98.7 \%$ in rural areas.

\section{Results}

Tree cover in the conterminous United States is estimated at $34.2 \%(\mathrm{SE}=0.2$ ) or 266.7 million hectares (659.0 million acres). Urban/community areas, which occupy $5.3 \%$ of the land area, have comparable tree cover at $35.1 \%(\mathrm{SE}=0.4)$ or 14.6 million hectares of tree cover (36.2 million acres) (Table 1). Including Alaska and 
Table

Summary of percent tree cover statewide, and within urban, community, urban/community (UC) and rural land.

\begin{tabular}{|c|c|c|c|c|c|c|c|c|c|c|c|c|c|c|c|c|c|c|c|}
\hline \multirow[b]{2}{*}{ State } & \multicolumn{15}{|c|}{$\underline{\text { Percent tree cover }}$} & \multirow[b]{2}{*}{$\% \mathrm{UC}^{\mathrm{a}}$} & \multirow{2}{*}{$\begin{array}{l}\% \text { of state tree cover } \\
\text { in UC }\end{array}$} & \multirow{2}{*}{$\begin{array}{l}\text { UC tree } \\
\text { cover (ha) }\end{array}$} & \multirow{2}{*}{$\begin{array}{l}\text { State tree } \\
\text { cover (ha) }\end{array}$} \\
\hline & Statewide & SE & $n$ & Urban & $\mathrm{SE}$ & $n$ & Comm. & SE & $n$ & UC & SE & $n$ & Rural & SE & $n$ & & & & \\
\hline Alabama & 70.0 & 1.5 & 1366 & 53.0 & 3.9 & 164 & 55.4 & 2.4 & 413 & 55.2 & 2.4 & 446 & 71.5 & 1.7 & 920 & 9.5 & 7.5 & 701,000 & $9,369,000$ \\
\hline Arizona & 19.2 & 0.7 & 3105 & 16.4 & 3.1 & 140 & 17.9 & 1.7 & 487 & 17.6 & 1.7 & 500 & 19.3 & 0.8 & 2605 & 5.3 & 4.9 & 277,000 & $5,659,000$ \\
\hline Arkansas & 57.2 & 1.6 & 1146 & 43.0 & 5.0 & 100 & 41.8 & 3.4 & 213 & 42.3 & 3.3 & 222 & 57.9 & 1.6 & 924 & 4.4 & 3.3 & 259,000 & $7,876,000$ \\
\hline California & 36.1 & 0.6 & 5835 & 19.9 & 1.5 & 728 & 26.3 & 1.3 & 1083 & 25.1 & 1.2 & 1237 & 37.1 & 0.6 & 4598 & 8.4 & 5.9 & 870,000 & $14,794,000$ \\
\hline Colorado & 23.6 & 0.8 & 2205 & 17.4 & 3.6 & 109 & 19.8 & 2.8 & 207 & 18.5 & 2.5 & 233 & 23.7 & 0.8 & 1972 & 2.6 & 2.0 & 130,000 & $6,353,000$ \\
\hline Connecticut & 72.6 & 2.8 & 298 & 66.5 & 3.6 & 173 & 66.0 & 4.7 & 100 & 67.4 & 3.4 & 190 & 75.9 & 4.1 & 108 & 38.8 & 36.0 & 337,000 & 938,000 \\
\hline Delaware & 33.3 & 4.0 & 200 & 38.0 & 4.9 & 100 & 33.0 & 4.7 & 100 & 35.0 & 4.8 & 100 & 33.0 & 4.7 & 100 & 17.2 & 18.1 & 32,000 & 175,000 \\
\hline Florida & 54.9 & 1.3 & 1991 & 35.1 & 2.0 & 553 & 33.9 & 1.9 & 654 & 35.5 & 1.7 & 829 & 58.4 & 1.5 & 1162 & 15.3 & 9.9 & 813,000 & $8,255,000$ \\
\hline Georgia & 66.4 & 1.3 & 1801 & 52.0 & 2.6 & 367 & 54.3 & 2.7 & 352 & 54.1 & 2.1 & 542 & 67.7 & 1.4 & 1259 & 9.5 & 7.7 & 781,000 & $10,128,000$ \\
\hline Idaho & 37.9 & 0.9 & 2127 & 13.0 & 3.4 & 100 & 12.0 & 3.2 & 100 & 10.0 & 3.0 & 100 & 38.2 & 1.0 & 2027 & 0.8 & 0.2 & 18,000 & $8,204,000$ \\
\hline Illinois & 15.6 & 1.1 & 1374 & 26.4 & 2.4 & 329 & 23.9 & 2.2 & 380 & 25.4 & 2.0 & 468 & 14.7 & 1.2 & 906 & 8.6 & 13.9 & 317,000 & $2,273,000$ \\
\hline Indiana & 25.7 & 1.5 & 1081 & 22.3 & 3.0 & 188 & 23.2 & 2.9 & 207 & 23.7 & 2.6 & 266 & 25.9 & 1.6 & 815 & 8.0 & 7.4 & 178,000 & $2,407,000$ \\
\hline Iowa & 10.4 & 0.9 & 1265 & 24.0 & 4.3 & 100 & 18.8 & 2.8 & 191 & 19.0 & 2.8 & 200 & 10.1 & 0.9 & 1065 & 3.6 & 6.6 & 100,000 & $1,511,000$ \\
\hline Kansas & 8.0 & 0.7 & 1483 & 28.0 & 4.5 & 100 & 26.5 & 4.4 & 102 & 25.0 & 4.0 & 116 & 7.7 & 0.7 & 1367 & 1.8 & 5.6 & 95,000 & $1,709,000$ \\
\hline Kentucky & 58.0 & 1.6 & 1081 & 26.9 & 4.3 & 104 & 19.6 & 3.2 & 153 & 22.1 & 3.1 & 181 & 60.0 & 1.7 & 900 & 5.1 & 1.9 & 117,000 & $6,072,000$ \\
\hline Louisiana & 51.5 & 2.0 & 906 & 32.2 & 3.8 & 152 & 35.2 & 3.1 & 236 & 34.9 & 2.9 & 278 & 52.6 & 2.1 & 628 & 6.2 & 4.2 & 265,000 & $6,313,000$ \\
\hline Maine & 83.1 & 1.3 & 834 & 54.0 & 5.0 & 100 & 51.6 & 4.5 & 122 & 52.3 & 4.4 & 128 & 84.4 & 1.4 & 706 & 4.0 & 2.5 & 176,000 & $7,016,000$ \\
\hline Maryland & 42.8 & 2.6 & 451 & 32.9 & 3.6 & 167 & 34.7 & 3.7 & 167 & 34.3 & 3.3 & 210 & 45.2 & 3.1 & 241 & 21.9 & 17.5 & 203,000 & $1,160,000$ \\
\hline Massachusetts & 70.8 & 2.3 & 472 & 64.5 & 3.0 & 251 & 60.9 & 3.8 & 161 & 65.1 & 2.7 & 304 & 74.4 & 3.4 & 168 & 38.6 & 35.5 & 534,000 & $1,507,000$ \\
\hline Michigan & 59.5 & 1.5 & 1492 & 34.6 & 2.8 & 289 & 34.0 & 2.9 & 262 & 35.0 & 2.5 & 377 & 61.4 & 1.6 & 1115 & 7.1 & 4.2 & 376,000 & $8,975,000$ \\
\hline Minnesota & 34.8 & 1.1 & 1788 & 31.0 & 3.7 & 158 & 33.8 & 2.4 & 379 & 34.0 & 2.4 & 400 & 34.9 & 1.1 & 1388 & 4.9 & 4.7 & 361,000 & $7,599,000$ \\
\hline Mississippi & 64.0 & 1.8 & 902 & 41.0 & 4.9 & 100 & 47.1 & 3.6 & 189 & 47.3 & 3.5 & 201 & 64.8 & 1.9 & 701 & 4.6 & 3.4 & 268,000 & $7,911,000$ \\
\hline Missouri & 40.3 & 1.4 & 1399 & 31.1 & 3.6 & 164 & 29.2 & 2.8 & 257 & 31.5 & 2.7 & 289 & 40.7 & 1.5 & 1110 & 4.6 & 3.6 & 263,000 & $7,272,000$ \\
\hline Montana & 27.5 & 0.7 & 3239 & 9.0 & 2.9 & 100 & 37.9 & 3.1 & 240 & 36.3 & 3.0 & 251 & 27.4 & 0.7 & 2988 & 2.0 & 2.7 & 279,000 & $10,478,000$ \\
\hline Nebraska & 3.6 & 0.5 & 1365 & 19.0 & 3.9 & 100 & 14.0 & 3.5 & 100 & 15.0 & 3.6 & 100 & 3.5 & 0.5 & 1265 & 1.0 & 4.0 & 29,000 & 714,000 \\
\hline Nevada & 11.6 & 0.7 & 2177 & 12.0 & 3.2 & 100 & 9.5 & 1.8 & 262 & 9.6 & 1.8 & 271 & 11.6 & 0.8 & 1906 & 2.7 & 2.3 & 75,000 & $3,313,000$ \\
\hline New Hampshire & 88.9 & 1.8 & 325 & 64.0 & 4.8 & 100 & 67.0 & 4.7 & 100 & 66.0 & 4.7 & 100 & 91.5 & 1.9 & 225 & 10.0 & 7.4 & 158,000 & $2,129,000$ \\
\hline New Jersey & 57.0 & 2.8 & 409 & 50.4 & 3.2 & 240 & 51.9 & 3.7 & 183 & 53.3 & 2.9 & 287 & 59.8 & 4.4 & 122 & 42.1 & 39.4 & 452,000 & $1,149,000$ \\
\hline New Mexico & 19.1 & 0.7 & 2576 & 12.0 & 3.2 & 100 & 12.9 & 2.6 & 163 & 12.0 & 2.5 & 175 & 19.2 & 0.7 & 2401 & 1.7 & 1.1 & 63,000 & $6,000,000$ \\
\hline New York & 65.0 & 1.1 & 1923 & 41.2 & 2.6 & 347 & 41.1 & 2.5 & 375 & 42.6 & 2.2 & 500 & 67.6 & 1.2 & 1423 & 10.4 & 6.8 & 561,000 & $8,248,000$ \\
\hline North Carolina & 62.6 & 1.2 & 1921 & 48.2 & 2.8 & 330 & 50.3 & 2.6 & 358 & 51.1 & 2.3 & 479 & 63.9 & 1.3 & 1442 & 10.0 & 8.1 & 663,000 & $8,156,000$ \\
\hline North Dakota & 2.6 & 0.4 & 1484 & 15.0 & 3.6 & 100 & 13.0 & 3.4 & 100 & 13.0 & 3.4 & 100 & 2.5 & 0.4 & 1384 & 0.9 & 4.4 & 21,000 & 476,000 \\
\hline Ohio & 39.9 & 1.2 & 1553 & 29.0 & 2.4 & 365 & 31.0 & 2.5 & 352 & 31.5 & 2.1 & 470 & 41.1 & 1.4 & 1083 & 12.4 & 9.8 & 419,000 & $4,274,000$ \\
\hline Oklahoma & 25.9 & 1.2 & 1555 & 18.9 & 3.5 & 127 & 31.5 & 2.2 & 447 & 31.2 & 2.2 & 455 & 25.5 & 1.3 & 1100 & 6.7 & 8.1 & 378,000 & $4,681,000$ \\
\hline Oregon & 40.8 & 0.9 & 2715 & 40.0 & 4.9 & 100 & 36.7 & 4.6 & 109 & 36.6 & 4.2 & 134 & 40.8 & 0.9 & 2581 & 1.5 & 1.4 & 141,000 & $10,235,000$ \\
\hline Pennsylvania & 65.8 & 1.2 & 1580 & 34.0 & 2.4 & 382 & 45.0 & 2.9 & 291 & 41.0 & 2.2 & 502 & 69.3 & 1.4 & 1078 & 12.2 & 7.6 & 590,000 & $7,727,000$ \\
\hline Rhode Island & 70.3 & 3.0 & 200 & 54.0 & 5.0 & 100 & 40.0 & 4.9 & 100 & 51.0 & 5.0 & 100 & 82.0 & 3.8 & 100 & 37.6 & 27.3 & 54,000 & 199,000 \\
\hline South Carolina & 64.6 & 1.6 & 1130 & 47.1 & 3.8 & 170 & 46.7 & 3.6 & 195 & 48.9 & 3.0 & 270 & 66.2 & 1.7 & 860 & 8.9 & 6.8 & 352,000 & $5,215,000$ \\
\hline South Dakota & 5.7 & 0.6 & 1371 & 21.0 & 4.1 & 100 & 13.0 & 3.4 & 100 & 14.0 & 3.5 & 100 & 5.7 & 0.6 & 1271 & 0.8 & 2.1 & 24,000 & $1,143,000$ \\
\hline Tennessee & 57.1 & 1.4 & 1511 & 39.2 & 3.4 & 212 & 45.2 & 2.7 & 330 & 43.8 & 2.5 & 388 & 58.6 & 1.6 & 1123 & 10.4 & 8.0 & 497,000 & $6,232,000$ \\
\hline Texas & 23.4 & 0.6 & 5314 & 32.0 & 1.8 & 666 & 31.3 & 1.4 & 1,086 & 31.4 & 1.3 & 1212 & 23.0 & 0.6 & 4102 & 4.9 & 6.6 & $1,058,000$ & $16,052,000$ \\
\hline Utah & 17.8 & 0.8 & 2441 & 15.0 & 3.6 & 100 & 16.2 & 2.6 & 204 & 16.4 & 2.6 & 207 & 17.8 & 0.8 & 2234 & 2.7 & 2.5 & 98,000 & $3,909,000$ \\
\hline Vermont & 81.5 & 2.2 & 412 & 53.0 & 5.0 & 100 & 51.0 & 5.0 & 100 & 53.0 & 5.0 & 100 & 82.3 & 2.2 & 312 & 2.8 & 1.8 & 36,000 & $2,015,000$ \\
\hline Virginia & 66.7 & 1.3 & 1540 & 34.8 & 3.2 & 224 & 38.6 & 2.8 & 306 & 39.8 & 2.5 & 372 & 69.6 & 1.4 & 1168 & 9.6 & 5.7 & 401,000 & $7,012,000$ \\
\hline Washington & 47.2 & 0.9 & 2198 & 32.8 & 3.3 & 198 & 34.1 & 3.0 & 255 & 34.6 & 2.7 & 321 & 47.8 & 0.9 & 1877 & 5.1 & 3.7 & 310,000 & $8,308,000$ \\
\hline West Virginia & 81.4 & 1.4 & 832 & 47.0 & 5.0 & 100 & 62.0 & 4.9 & 100 & 61.0 & 4.9 & 100 & 82.3 & 1.5 & 732 & 4.1 & 3.1 & 156,000 & $5,110,000$ \\
\hline Wisconsin & 47.7 & 1.4 & 1403 & 29.2 & 3.8 & 144 & 30.9 & 2.9 & 262 & 31.8 & 2.7 & 305 & 48.7 & 1.5 & 1098 & 5.4 & 3.6 & 250,000 & $6,927,000$ \\
\hline Wyoming & 14.5 & 0.7 & 2322 & 9.0 & 2.9 & 100 & 20.5 & 3.0 & 176 & 19.9 & 3.0 & 181 & 14.4 & 0.7 & 2141 & 1.9 & 2.6 & 96,000 & $3,670,000$ \\
\hline
\end{tabular}




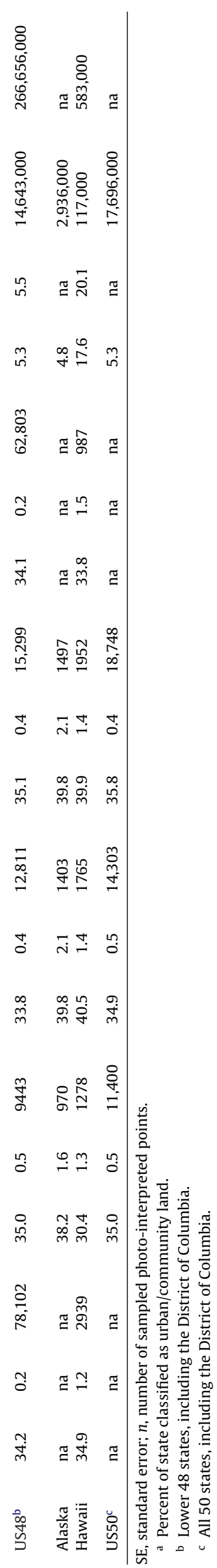

Hawaii, national tree cover in urban/community areas is estimated at $35.8 \%(\mathrm{SE}=0.4)$ or 17.7 million hectares of tree cover $(43.7$ million acres). Statewide, percent tree cover is highest in New Hampshire $(88.9 \%, \mathrm{SE}=1.8)$ and lowest in North Dakota $(2.6 \%, \mathrm{SE}=0.4)$. In urban/community areas, percent tree cover is highest in Connecticut $(67.4 \%, \mathrm{SE}=3.4)$ and lowest in Nevada $(9.6 \%, \mathrm{SE}=1.8)$ (Table 1$)$.

Impervious cover in the conterminous United States is estimated at $2.4 \%(\mathrm{SE}=0.1)$ or 18.4 million hectares of impervious cover (45.5 million acres). In contrast, urban/community areas have higher percent impervious cover at $17.5 \%(\mathrm{SE}=0.3)$ or 7.3 million hectares of impervious cover (18.0 million acres) (Table 2). Including Alaska and Hawaii, national impervious cover in urban/community areas is estimated at $14.9 \%(\mathrm{SE}=0.3)$ or 7.4 million hectares of impervious cover (18.2 million acres). State wide, percent impervious cover is highest in New Jersey $(12.1 \%, \mathrm{SE}=1.6)$ and lowest in Wyoming $(0.5 \%, \mathrm{SE}=0.1)$. In urban/community areas, percent impervious cover is highest in Nebraska $(32.0 \%, \mathrm{SE}=4.7)$ and lowest in Wyoming $(2.2 \%, \mathrm{SE}=1.1)$ (Table 2$)$.

Overall, there was no statistical difference between urban/community percent tree cover and rural percent tree cover, but urban/community areas did have significantly higher percent impervious cover (+16.0\%) (Table 3$)$. However, differences in percent tree cover did vary by state, with nine states having significantly higher percent tree cover in urban/community areas than rural areas, and 26 states having significantly lower percent tree cover. In terms of impervious surfaces, all states showed an increase in percent impervious cover in urban/community land versus rural land in the state, with 46 having a statistically significant increase (Table 3 ).

Tree cover per capita in urban areas averaged $377 \mathrm{~m}^{2}$ and was greatest in New Hampshire (1266) and lowest in Nevada (92). Impervious cover per capita averaged $274 \mathrm{~m}^{2}$ and was greatest in Idaho (444) and lowest in New York (167) (Table 4). For the conterminous United States, tree cover per capita was $9512 \mathrm{~m}^{2}$ and impervious cover per capita was $657 \mathrm{~m}^{2}$.

Percent tree cover within urban areas and urban/community areas was correlated with overall state percent tree cover (Spearman correlation; $r=0.87$ and $r=0.89$, respectively). Percent tree cover in urban areas was negatively correlated with urban population density (Spearman correlation: $r=-0.43$ ), while percent impervious cover was positively correlated with urban population density (Spearman correlation: $r=0.67$ ). Urban tree and urban impervious cover were negatively correlated (Spearman correlation: $r=-0.72$ ).

\section{Discussion}

\subsection{Tree cover}

Tree cover in the conterminous United States is an important landscape element, covering over $1 / 3$ of the nation and occupying 266.7 million hectares. Even within urban lands where people and impervious surfaces are concentrated, tree cover is still a dominant element, covering approximately the same percent of land cover (35.0\%). A previous urban tree cover study for the conterminous United States, based on advanced very high resolution radiometer (AVHRR) data and Landsat thematic mapper data (Zhu, 1994), estimated percent urban tree cover at $27 \%$, with urban tree cover highest in forested regions (34.4\%), followed by grassland areas (17.8\%) and deserts (9.3\%) (Nowak, Noble, Sisinni, \& Dwyer, 2001). However this estimate was likely conservative based on the limitations of the AVHRR data (Dwyer et al., 2000).

Statewide tree cover varies, as expected, based on the local environment (e.g., precipitation, temperature, natural vegetation types). Tree cover within urban areas was correlated with overall 
Table 2

Summary of percent impervious cover statewide, and within urban, community, and urban/community (UC) land.

\begin{tabular}{|c|c|c|c|c|c|c|c|c|c|c|c|c|c|c|c|c|c|c|c|}
\hline \multirow[b]{2}{*}{ State } & \multicolumn{15}{|c|}{ Percent impervious cover } & \multirow[b]{2}{*}{$\% \mathrm{UC}^{\mathrm{a}}$} & \multirow{2}{*}{$\begin{array}{l}\text { \% of state imp. cover } \\
\text { in UC }\end{array}$} & \multirow{2}{*}{$\begin{array}{l}\text { UC imp. } \\
\text { cover (ha) }\end{array}$} & \multirow{2}{*}{$\begin{array}{l}\text { State imp } \\
\text { cover (ha) }\end{array}$} \\
\hline & Statewide & SE & $n$ & Urban & SE & $n$ & Comm. & SE & $n$ & UC & $\mathrm{SE}$ & $n$ & Rural & SE & $n$ & & & & \\
\hline Alabama & 2.7 & 0.5 & 1366 & 15.2 & 2.8 & 164 & 8.2 & 1.4 & 413 & 8.3 & 1.3 & 446 & 2.1 & 0.5 & 920 & 9.5 & 28.8 & 105,000 & 366,000 \\
\hline Arizona & 1.3 & 0.2 & 3105 & 37.9 & 4.1 & 140 & 13.3 & 1.5 & 487 & 13.8 & 1.5 & 500 & 0.6 & 0.2 & 2605 & 5.3 & 57.1 & 217,000 & 380,000 \\
\hline Arkansas & 2.5 & 0.5 & 1146 & 25.0 & 4.3 & 100 & 15.5 & 2.5 & 213 & 14.9 & 2.4 & 222 & 1.9 & 0.5 & 924 & 4.4 & 26.8 & 91,000 & 340,000 \\
\hline California & 2.9 & 0.2 & 5835 & 39.8 & 1.8 & 728 & 28.5 & 1.4 & 1083 & 26.9 & 1.3 & 1237 & 0.7 & 0.1 & 4598 & 8.4 & 77.1 & 931,000 & $1,207,000$ \\
\hline Colorado & 1.4 & 0.2 & 2205 & 28.4 & 4.3 & 109 & 17.4 & 2.6 & 207 & 17.2 & 2.5 & 233 & 1.0 & 0.2 & 1972 & 2.6 & 31.1 & 121,000 & 390,000 \\
\hline Connecticut & 7.7 & 1.6 & 298 & 11.6 & 2.4 & 173 & 12.0 & 3.2 & 100 & 11.1 & 2.3 & 190 & 5.6 & 2.2 & 108 & 38.8 & 55.8 & 55,000 & 99,000 \\
\hline Delaware & 6.2 & 1.7 & 200 & 19.0 & 3.9 & 100 & 21.0 & 4.1 & 100 & 17.0 & 3.8 & 100 & 4.0 & 2.0 & 100 & 17.2 & 46.9 & 15,000 & 33,000 \\
\hline Florida & 6.9 & 0.6 & 1991 & 27.5 & 1.9 & 553 & 22.2 & 1.6 & 654 & 21.5 & 1.4 & 829 & 4.3 & 0.7 & 1162 & 15.3 & 47.3 & 492,000 & $1,041,000$ \\
\hline Georgia & 3.1 & 0.4 & 1801 & 17.7 & 2.0 & 367 & 13.6 & 1.8 & 352 & 13.5 & 1.5 & 542 & 2.0 & 0.4 & 1259 & 9.5 & 40.8 & 194,000 & 476,000 \\
\hline Idaho & 1.1 & 0.2 & 2127 & 37.0 & 4.8 & 100 & 22.0 & 4.1 & 100 & 23.0 & 4.2 & 100 & 0.9 & 0.2 & 2027 & 0.8 & 17.3 & 41,000 & 238,000 \\
\hline Illinois & 4.8 & 0.5 & 1374 & 30.7 & 2.5 & 329 & 30.8 & 2.4 & 380 & 26.1 & 2.0 & 468 & 2.8 & 0.6 & 906 & 8.6 & 46.2 & 325,000 & 702,000 \\
\hline Indiana & 3.7 & 0.5 & 1081 & 25.5 & 3.2 & 188 & 25.6 & 3.0 & 207 & 22.6 & 2.6 & 266 & 2.0 & 0.5 & 815 & 8.0 & 49.5 & 170,000 & 343,000 \\
\hline Iowa & 3.0 & 0.5 & 1265 & 27.0 & 4.4 & 100 & 20.4 & 2.9 & 191 & 19.5 & 2.8 & 200 & 2.4 & 0.5 & 1065 & 3.6 & 23.6 & 103,000 & 434,000 \\
\hline Kansas & 2.2 & 0.4 & 1483 & 23.0 & 4.2 & 100 & 22.5 & 4.1 & 102 & 19.8 & 3.7 & 116 & 1.8 & 0.4 & 1367 & 1.8 & 16.4 & 76,000 & 461,000 \\
\hline Kentucky & 2.3 & 0.5 & 1081 & 16.3 & 3.6 & 104 & 12.4 & 2.7 & 153 & 12.2 & 2.4 & 181 & 1.8 & 0.5 & 900 & 5.1 & 26.9 & 64,000 & 239,000 \\
\hline Louisiana & 2.9 & 0.6 & 906 & 19.1 & 3.2 & 152 & 13.1 & 2.2 & 236 & 12.2 & 2.0 & 278 & 2.3 & 0.6 & 628 & 6.2 & 26.1 & 93,000 & 356,000 \\
\hline Maine & 3.2 & 0.6 & 834 & 19.0 & 3.9 & 100 & 13.1 & 3.1 & 122 & 12.5 & 2.9 & 128 & 2.8 & 0.6 & 706 & 4.0 & 15.5 & 42,000 & 272,000 \\
\hline Maryland & 6.1 & 1.0 & 451 & 21.6 & 3.2 & 167 & 21.6 & 3.2 & 167 & 19.0 & 2.7 & 210 & 2.4 & 1.0 & 241 & 21.9 & 68.6 & 113,000 & 164,000 \\
\hline Massachusetts & 7.4 & 1.1 & 472 & 16.7 & 2.4 & 251 & 16.1 & 2.9 & 161 & 14.5 & 2.0 & 304 & 3.0 & 1.3 & 168 & 38.6 & 75.3 & 119,000 & 158,000 \\
\hline Michigan & 4.1 & 0.5 & 1492 & 31.5 & 2.7 & 289 & 29.0 & 2.8 & 262 & 26.8 & 2.3 & 377 & 2.3 & 0.5 & 1115 & 7.1 & 47.0 & 288,000 & 612,000 \\
\hline Minnesota & 2.2 & 0.3 & 1788 & 24.1 & 3.4 & 158 & 13.2 & 1.7 & 379 & 13.3 & 1.7 & 400 & 1.7 & 0.3 & 1388 & 4.9 & 29.1 & 141,000 & 483,000 \\
\hline Mississippi & 3.6 & 0.7 & 902 & 17.0 & 3.8 & 100 & 12.7 & 2.4 & 189 & 11.9 & 2.3 & 201 & 3.2 & 0.7 & 701 & 4.6 & 15.0 & 68,000 & 450,000 \\
\hline Missouri & 2.4 & 0.4 & 1399 & 22.0 & 3.2 & 164 & 18.3 & 2.4 & 257 & 18.0 & 2.3 & 289 & 1.6 & 0.4 & 1110 & 4.6 & 34.8 & 150,000 & 431,000 \\
\hline Montana & 0.7 & 0.1 & 3239 & 28.0 & 4.5 & 100 & 8.3 & 1.8 & 240 & 8.4 & 1.7 & 251 & 0.6 & 0.1 & 2988 & 2.0 & 22.7 & 64,000 & 284,000 \\
\hline Nebraska & 1.6 & 0.3 & 1365 & 28.0 & 4.5 & 100 & 33.0 & 4.7 & 100 & 32.0 & 4.7 & 100 & 1.3 & 0.3 & 1265 & 1.0 & 19.7 & 62,000 & 316,000 \\
\hline Nevada & 0.6 & 0.1 & 2177 & 49.0 & 5.0 & 100 & 8.8 & 1.7 & 262 & 9.2 & 1.8 & 271 & 0.4 & 0.1 & 1906 & 2.7 & 41.2 & 72,000 & 176,000 \\
\hline New Hampshire & 5.0 & 1.3 & 325 & 18.0 & 3.8 & 100 & 9.0 & 3.5 & 67 & 12.0 & 3.2 & 100 & 4.2 & 1.4 & 225 & 10.0 & 23.9 & 29,000 & 120,000 \\
\hline New Jersey & 12.1 & 1.6 & 409 & 22.5 & 2.7 & 240 & 21.9 & 3.1 & 183 & 19.9 & 2.4 & 287 & 6.5 & 2.1 & 122 & 42.1 & 69.0 & 169,000 & 244,000 \\
\hline New Mexico & 1.0 & 0.2 & 2576 & 24.0 & 4.3 & 100 & 14.7 & 2.8 & 163 & 15.4 & 2.7 & 175 & 0.7 & 0.2 & 2401 & 1.7 & 26.1 & 81,000 & 312,000 \\
\hline New York & 4.5 & 0.4 & 1923 & 27.4 & 2.4 & 347 & 24.3 & 2.2 & 375 & 22.4 & 1.9 & 500 & 2.4 & 0.4 & 1423 & 10.4 & 51.5 & 295,000 & 573,000 \\
\hline North Carolina & 4.9 & 0.5 & 1921 & 18.5 & 2.1 & 330 & 17.3 & 2.0 & 358 & 15.7 & 1.7 & 479 & 3.7 & 0.5 & 1442 & 10.0 & 32.1 & 203,000 & 633,000 \\
\hline North Dakota & 1.1 & 0.3 & 1484 & 26.0 & 4.4 & 100 & 10.0 & 3.0 & 100 & 10.0 & 3.0 & 100 & 1.0 & 0.3 & 1384 & 0.9 & 7.9 & 16,000 & 204,000 \\
\hline Ohio & 5.5 & 0.5 & 1553 & 27.1 & 2.3 & 365 & 28.1 & 2.4 & 352 & 24.5 & 2.0 & 470 & 2.8 & 0.5 & 1083 & 12.4 & 55.2 & 326,000 & 590,000 \\
\hline Oklahoma & 2.7 & 0.4 & 1555 & 30.7 & 4.1 & 127 & 13.0 & 1.6 & 447 & 12.7 & 1.6 & 455 & 2.0 & 0.5 & 1100 & 6.7 & 31.2 & 155,000 & 496,000 \\
\hline Oregon & 0.8 & 0.1 & 2715 & 26.0 & 4.4 & 100 & 22.0 & 4.0 & 109 & 19.4 & 3.4 & 134 & 0.5 & 0.1 & 2581 & 1.5 & 36.8 & 75,000 & 203,000 \\
\hline Pennsylvania & 4.6 & 0.5 & 1580 & 24.6 & 2.2 & 382 & 18.6 & 2.3 & 291 & 19.1 & 1.8 & 502 & 2.5 & 0.5 & 1078 & 12.2 & 51.3 & 275,000 & 536,000 \\
\hline Rhode Island & 10.9 & 2.0 & 200 & 26.0 & 4.4 & 100 & 36.0 & 4.8 & 100 & 24.0 & 4.3 & 100 & 3.0 & 1.9 & 100 & 37.6 & 82.8 & 26,000 & 31,000 \\
\hline South Carolina & 4.4 & 0.6 & 1130 & 17.1 & 2.9 & 170 & 15.4 & 2.6 & 195 & 14.1 & 2.1 & 270 & 3.5 & 0.7 & 860 & 8.9 & 28.3 & 101,000 & 359,000 \\
\hline South Dakota & 1.7 & 0.4 & 1371 & 33.0 & 4.7 & 100 & 18.0 & 3.8 & 100 & 18.0 & 3.8 & 100 & 1.6 & 0.4 & 1271 & 0.8 & 9.0 & 30,000 & 338,000 \\
\hline Tennessee & 3.4 & 0.4 & 1511 & 19.3 & 2.7 & 212 & 15.8 & 2.0 & 330 & 14.7 & 1.8 & 388 & 2.0 & 0.4 & 1123 & 10.4 & 45.4 & 167,000 & 367,000 \\
\hline Texas & 2.0 & 0.2 & 5314 & 25.1 & 1.7 & 666 & 16.4 & 1.1 & 1086 & 16.5 & 1.1 & 1212 & 1.2 & 0.2 & 4102 & 4.9 & 41.5 & 556,000 & $1,340,000$ \\
\hline Utah & 0.9 & 0.2 & 2441 & 36.0 & 4.8 & 100 & 11.3 & 2.2 & 204 & 11.6 & 2.2 & 207 & 0.6 & 0.2 & 2234 & 2.7 & 34.2 & 69,000 & 201,000 \\
\hline Vermont & 1.9 & 0.6 & 412 & 22.0 & 4.1 & 100 & 20.0 & 4.0 & 100 & 17.0 & 3.8 & 100 & 1.4 & 0.7 & 312 & 2.8 & 25.4 & 12,000 & 46,000 \\
\hline Virginia & 4.3 & 0.5 & 1540 & 21.9 & 2.8 & 224 & 17.3 & 2.2 & 306 & 16.1 & 1.9 & 372 & 3.0 & 0.5 & 1168 & 9.6 & 36.3 & 163,000 & 448,000 \\
\hline Washington & 1.6 & 0.2 & 2198 & 26.3 & 3.1 & 198 & 19.2 & 2.5 & 255 & 18.1 & 2.1 & 321 & 0.7 & 0.2 & 1877 & 5.1 & 56.5 & 162,000 & 287,000 \\
\hline West Virginia & 2.0 & 0.5 & 832 & 20.0 & 4.0 & 100 & 14.0 & 3.5 & 100 & 12.0 & 3.2 & 100 & 1.6 & 0.5 & 732 & 4.1 & 24.6 & 31,000 & 128,000 \\
\hline Wisconsin & 2.8 & 0.4 & 1403 & 22.2 & 3.5 & 144 & 15.6 & 2.2 & 262 & 14.8 & 2.0 & 305 & 2.1 & 0.4 & 1098 & 5.4 & 28.5 & 116,000 & 406,000 \\
\hline
\end{tabular}




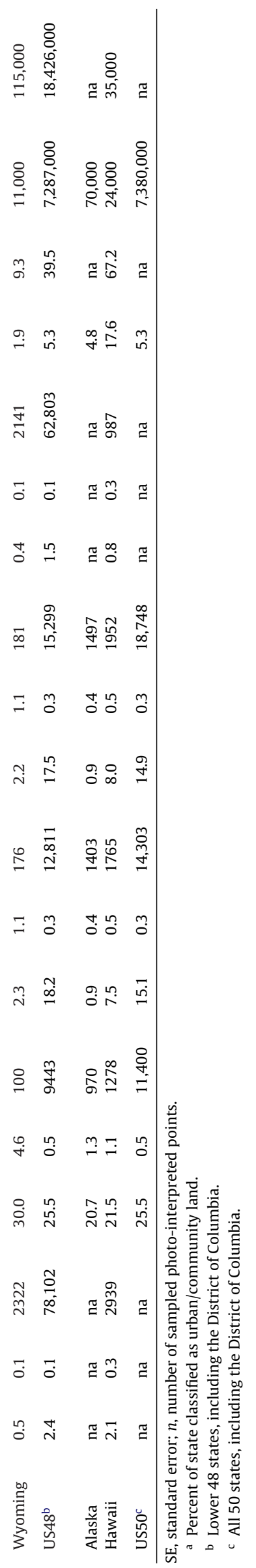

Table 3

Difference in percent tree and impervious cover between urban/community areas and rural areas by state (urban/community cover minus rural cover - positive values indicate greater cover in urban/community areas).

\begin{tabular}{|c|c|c|c|}
\hline State & Tree cover ${ }^{\mathrm{a}}$ & State & Impervious cover ${ }^{\mathrm{b}}$ \\
\hline Kansas & $17.3^{*}$ & Nebraska & $30.7^{*}$ \\
\hline Nebraska & $11.5^{*}$ & California & $26.2^{*}$ \\
\hline Illinois & $10.7^{*}$ & Michigan & $24.5^{*}$ \\
\hline North Dakota & $10.5^{*}$ & Illinois & $23.2^{*}$ \\
\hline Iowa & $8.9^{*}$ & Idaho & $22.1^{*}$ \\
\hline Montana & $8.9^{*}$ & Ohio & $21.6^{*}$ \\
\hline Texas & $8.5^{*}$ & Rhode Island & $21.0^{*}$ \\
\hline South Dakota & $8.3^{*}$ & Indiana & $20.5^{*}$ \\
\hline Oklahoma & 5.7 & New York & $20.0^{*}$ \\
\hline Wyoming & 5.5 & Oregon & $18.9^{*}$ \\
\hline Delaware & 2.0 & Kansas & $18.0^{*}$ \\
\hline Minnesota & -0.9 & Washington & $17.3^{*}$ \\
\hline Utah & -1.4 & Florida & $17.2^{*}$ \\
\hline Arizona & -1.7 & Iowa & $17.1^{*}$ \\
\hline Nevada & -2.0 & Maryland & $16.6^{*}$ \\
\hline Indiana & -2.2 & Pennsylvania & $16.6^{*}$ \\
\hline Oregon & -4.3 & South Dakota & $16.4^{*}$ \\
\hline Colorado & -5.3 & Missouri & $16.4^{*}$ \\
\hline New Jersey & -6.5 & Colorado & $16.1^{*}$ \\
\hline New Mexico & $-7.2^{*}$ & Vermont & $15.6^{*}$ \\
\hline Connecticut & -8.6 & Texas & $15.3^{*}$ \\
\hline Missouri & $-9.2^{*}$ & New Mexico & $14.7^{*}$ \\
\hline Massachusetts & -9.3 & New Jersey & $13.4^{*}$ \\
\hline Ohio & $-9.6^{*}$ & Arizona & $13.2^{*}$ \\
\hline Maryland & -10.9 & Virginia & $13.1^{*}$ \\
\hline California & $-12.0^{*}$ & Delaware & $13.0^{*}$ \\
\hline North Carolina & $-12.7^{*}$ & Arkansas & $13.0^{*}$ \\
\hline Washington & $-13.2^{*}$ & Tennessee & $12.6^{*}$ \\
\hline Georgia & $-13.6^{*}$ & Wisconsin & $12.6^{*}$ \\
\hline Tennessee & $-14.8^{*}$ & North Carolina & $12.0^{*}$ \\
\hline Arkansas & $-15.5^{*}$ & Minnesota & $11.6^{*}$ \\
\hline Alabama & $-16.4^{*}$ & Massachusetts & $11.5^{*}$ \\
\hline Wisconsin & $-16.9^{*}$ & Georgia & $11.4^{*}$ \\
\hline South Carolina & $-17.3^{*}$ & Utah & $11.0^{*}$ \\
\hline Mississippi & $-17.6^{*}$ & Oklahoma & $10.7^{*}$ \\
\hline Louisiana & $-17.7^{*}$ & South Carolina & $10.6^{*}$ \\
\hline West Virginia & $-21.3^{*}$ & Kentucky & $10.4^{*}$ \\
\hline Florida & $-23.0^{*}$ & West Virginia & $10.4^{*}$ \\
\hline New York & $-25.0^{*}$ & Louisiana & $9.9^{*}$ \\
\hline New Hampshire & $-25.5^{*}$ & Maine & $9.7^{*}$ \\
\hline Michigan & $-26.3^{*}$ & North Dakota & $9.0^{*}$ \\
\hline Idaho & $-28.2^{*}$ & Nevada & $8.9^{*}$ \\
\hline Pennsylvania & $-28.3^{*}$ & Mississippi & $8.7^{*}$ \\
\hline Vermont & $-29.3^{*}$ & Montana & $7.8^{*}$ \\
\hline Virginia & $-29.8^{*}$ & New Hampshire & 7.8 \\
\hline Rhode Island & $-31.0^{*}$ & Alabama & $6.1^{*}$ \\
\hline Maine & $-32.1^{*}$ & Connecticut & 5.5 \\
\hline Kentucky & $-37.9^{*}$ & Wyoming & 1.8 \\
\hline US48 & 1.0 & US48 & $16.0^{*}$ \\
\hline Alaska & na & Alaska & na \\
\hline Hawaii & $6.1^{*}$ & Hawaii & $7.2^{*}$ \\
\hline
\end{tabular}

a Difference in tree cover (urban/community cover - rural cover).

b Difference in impervious cover (urban/community cover - rural cover).

c Lower 48 states, including the District of Columbia.

* Significant difference among 95\% confidence intervals.

state tree cover and negatively correlated with urban population density. Connecticut, Massachusetts and New Hampshire have greater than $60 \%$ tree cover in both their urban and community lands. These states have high percent tree cover naturally and have below average urban population densities (U.S. urban population density $=931$ people $\left./ \mathrm{km}^{2} ; \mathrm{CT}=655 ; \mathrm{MA}=802 ; \mathrm{NH}=506\right)$. Thus the naturally forested environment in conjunction lower urban population densities can help enhance percent tree canopy cover. Other factors, such as management programs to enhance tree cover in urban areas also likely play a role in sustaining urban tree cover in these states. The lower population densities may also lead to lower 
Table 4

Tree and impervious cover $\left(\mathrm{m}^{2}\right)$ per capita in urban areas by state.

\begin{tabular}{|c|c|c|c|}
\hline \multicolumn{2}{|l|}{ Tree cover } & \multicolumn{2}{|l|}{ Impervious cover } \\
\hline State & $\mathrm{m}^{2} /$ capita & State & $\mathrm{m}^{2} /$ capita \\
\hline New Hampshire & 1266 & Idaho & 444 \\
\hline Connecticut & 1015 & Arkansas & 415 \\
\hline Alabama & 988 & Wyoming & 408 \\
\hline Maine & 959 & Oklahoma & 406 \\
\hline South Carolina & 928 & Montana & 386 \\
\hline North Carolina & 912 & Nevada & 377 \\
\hline Georgia & 854 & Michigan & 365 \\
\hline Vermont & 852 & South Dakota & 365 \\
\hline Massachusetts & 804 & Arizona & 363 \\
\hline West Virginia & 804 & Vermont & 360 \\
\hline Arkansas & 714 & New Hampshire & 356 \\
\hline Mississippi & 698 & North Carolina & 350 \\
\hline Tennessee & 678 & West Virginia & 349 \\
\hline Rhode Island & 565 & New Mexico & 346 \\
\hline Delaware & 472 & Maine & 337 \\
\hline New Jersey & 459 & South Carolina & 336 \\
\hline Louisiana & 423 & Indiana & 336 \\
\hline Michigan & 401 & Tennessee & 335 \\
\hline Pennsylvania & 395 & Utah & 326 \\
\hline Virginia & 395 & Iowa & 321 \\
\hline Oregon & 391 & Ohio & 317 \\
\hline Florida & 391 & Florida & 306 \\
\hline Missouri & 376 & Washington & 297 \\
\hline Kentucky & 374 & Mississippi & 297 \\
\hline Washington & 371 & Georgia & 291 \\
\hline Minnesota & 345 & Pennsylvania & 285 \\
\hline Texas & 342 & Alabama & 284 \\
\hline Ohio & 340 & Nebraska & 278 \\
\hline Maryland & 338 & Rhode Island & 272 \\
\hline Wisconsin & 334 & North Dakota & 270 \\
\hline Kansas & 323 & Texas & 268 \\
\hline Indiana & 294 & Minnesota & 267 \\
\hline Iowa & 273 & Kansas & 266 \\
\hline New York & 252 & Missouri & 265 \\
\hline Oklahoma & 250 & Illinois & 259 \\
\hline South Dakota & 232 & California & 255 \\
\hline Illinois & 223 & Wisconsin & 255 \\
\hline Nebraska & 179 & Oregon & 254 \\
\hline New Mexico & 173 & Colorado & 254 \\
\hline Idaho & 160 & Louisiana & 250 \\
\hline Arizona & 158 & Virginia & 248 \\
\hline Colorado & 156 & Delaware & 236 \\
\hline North Dakota & 156 & Kentucky & 227 \\
\hline Utah & 136 & Maryland & 221 \\
\hline California & 128 & Massachusetts & 209 \\
\hline Montana & 124 & New Jersey & 205 \\
\hline Wyoming & 122 & Connecticut & 176 \\
\hline Nevada & 92 & New York & 167 \\
\hline US $48^{a}$ & 377 & US48 & 274 \\
\hline Alaska & 642 & Alaska & 348 \\
\hline Hawaii & 260 & Hawaii & 189 \\
\hline US50 ${ }^{\mathrm{b}}$ & 377 & US50 ${ }^{\mathrm{b}}$ & 274 \\
\hline
\end{tabular}

a Lower 48 states, including the District of Columbia.

b All 50 states, including the District of Columbia.

percent impervious cover (Table 2 ) and allow more space available for natural regeneration in these areas.

Dominant factors that affect urban tree cover are surrounding natural environment and land-use distribution (Nowak et al., 1996). The surrounding natural environment has a substantial influence as it can provide seed sources for new trees and an environment that may or may not be conducive to tree establishment and growth. The land use patterns determine the amount of potential available space for trees (non-impervious cover) and the type of management, planting or natural regeneration that may occur. Thus the mix of tree and impervious surfaces in urban areas is influenced by the social environment in the context of the natural environment.
Natural regeneration can play an important role in urban tree cover. In forested regions where natural regeneration readily occurs, human actions tend to prohibit tree cover through such actions as constructing impervious surfaces, soil compaction, and mowing of lawns. The reduction of these actions could increase tree cover in urban areas within naturally forested regions. Managers often supplement natural regeneration with tree planting and maintenance to sustain urban tree canopy in the United States.

However, in areas where natural regeneration is not common (i.e., grassland and desert areas), much of the urban tree cover comes through human actions of planting and maintenance. The percentage of the urban tree population that is planted is greater in cities developed in grassland areas as compared to cities developed in forests and tends to increase with increased population density and percent impervious cover in cities (Nowak, 2012). Enhancing tree cover in environments that tend to be precipitation limited involves various costs, both economic and environmental. Planting trees in these environments can produce substantial benefits for the urban population, but often require water or economic resources that may be scarce.

\subsection{Urban vs. rural tree cover}

Urban/community areas showed various differences in tree cover relative to the rural land in the states. In predominantly grassland states, urban/community development tends to increase tree cover, while in forested states it tends to decrease tree cover. In more desert states, tree cover did not change much or only declined slightly. Thus urban development has the potential to increase tree cover as population densities increase in some areas.

Some of the differences in tree cover between rural and urban/community lands may be associated with differences in natural vegetation types across a state (e.g., most states are not entirely forested) and locations of cities within a state. As cities are often located near water sources (e.g., rivers), cities in predominantly grassland states may be developed in areas with naturally higher tree cover (e.g., riparian zones) than more rural areas. Thus some of the differences associated with relatively higher tree cover in urban/community vs. rural lands in grassland states may be due to location, rather than human-influenced tree cover processes (e.g., tree planting) associated with urbanization. Also, agriculture is a dominant land use in rural lands in grassland states, and agricultural processes tend to limit tree cover in rural areas of these states.

\subsection{Impervious cover}

Impervious cover, as expected, is a less dominant cover type nationally ( $2.4 \%$ or 18.4 million hectares), but increases in dominance in urban areas (25.5\%). The ratio of tree to impervious cover is about $14: 1$ nationally, but only about $1.4: 1$ within urban areas, a 10 fold difference. There were no substantial differences in impervious cover by natural vegetation types, but differences were exhibited based on population density. States with more densely populated areas, typically in the Northeast, tended to have higher percent impervious surfaces. States with greater than 6\% impervious surfaces were New Jersey (12.1), Rhode Island (10.9), Connecticut (7.7), Massachusetts (7.4), Florida (6.9), Delaware (6.2) and Maryland (6.1).

Percent urban impervious cover averaged $25 \%$, but can be higher at the city level in densely populated areas. Impervious cover in 20 U.S. cities averaged $43 \%$ and was as high as $61.1 \%$ in New York City (Nowak \& Greenfield, 2012). Within urban areas, impervious cover was over $30 \%$ in 10 states and was a low as $11.6 \%$ in Connecticut. One reason for the relatively low estimate of impervious cover in Connecticut could be the high percent tree cover within urban Connecticut (66.5\%). Interpreters conducted dual classifications at each 
point (tree and impervious), but in heavily tree covered areas, the interpreter may not be able to see what is under the tree canopy. Percent impervious cover in urban areas was negatively correlated with percent urban tree cover. Thus, impervious cover estimates are likely conservative, particularly in more heavily tree covered areas.

One potential approach to improve estimation of impervious surface cover is to use leaf-off imagery. Various satellite-based approaches have estimated impervious surface for the United States, but likely underestimate impervious cover. NLCD estimates impervious cover in the conterminous United States at about 1.3\%, while another satellite-based study estimated it at $1.1 \%$ (Elvidge et al., 2007). Both of these satellite-based estimates have lower estimates than found in this photo-interpretation-based study $(2.4 \%$ impervious cover). Part of the reason for this difference may be due to the time period between estimates. Both satellite-based studies were based on data circa 2000, while this study's imagery was circa 2005. Thus new impervious surfaces constructed between 2000 and 2005 could cause part of the difference in estimation, but likely not all. It is possible that satellite-derived estimates have limitations in the estimation of impervious cover that may also be related to tree cover (leaves, branches, shadows) obscuring impervious cover or the relatively coarse resolution of the imagery ( $30 \mathrm{~m}$ to $1 \mathrm{~km}$ pixel resolution). As photo-interpreters were asked to record impervious cover beneath tree canopies by looking for clues of impervious cover (e.g., obvious extensions of roads, buildings, parking lots beneath tree cover), there is a chance that interpreters overestimated impervious cover in these cases as they could not directly see beneath the tree canopies. However, it is more likely that interpreters underestimated impervious cover.

Though a relatively small element of cover nationwide, impervious cover can be a significant element in urban areas and significantly affect air temperatures, energy use, water quality and stream flow, and consequently human health and well-being in cities. Percent impervious cover is related to local population density, but various designs can help minimize the environmental impacts of impervious surfaces while still providing the necessary transportation and building needs of an urban population (e.g., pervious paving, green roofs). One of the keys in developing the urban landscape is to understand the social and natural environments and then design and manage with and within nature to sustain optimal benefits for the urban residents, while minimizing costs (e.g., McHarg, 1992).

Urban land, where relatively high densities of people reside, and community land, which is defined by political boundaries, had similar results in relation to tree cover. However, community areas had significantly lower percent impervious cover ( 25.5 vs. $18.2 \%$ ). This lower percent impervious cover is likely due to lower population densities in the community areas (urban population density $=931$ people $/ \mathrm{km}^{2}$; community population density $=594$ people $/ \mathrm{km}^{2}$ ), and therefore less associated development. Impervious cover per capita tended to be highest in states with lowest population densities, indicating that percent impervious cover in urban areas likely increases at slower rates than population density increases. That is, though population density is correlated with impervious surfaces, there is likely some threshold of impervious cover where this cover does not need to increase much to sustain increasingly higher population densities.

\subsection{Optimal tree cover}

One of the key questions in urban landscape design is what is the optimal mix of tree and impervious cover? The answer to this question depends upon a number of factors, including natural vegetation cover, the costs and benefits associated with tree cover in the region, population density, and the interests/needs of local community members. Optimal tree cover in forested regions will likely be higher than in grasslands or deserts. Determining the true optimal tree cover for an area would depend upon a more in-depth analysis of costs and benefits derived from tree cover and community interests in a region, and have not been quantified yet for any region. However, setting of minimal goals for tree canopy cover can facilitate standards and management plans to sustain tree cover and associated environmental services in urban and urbanizing areas.

The data illustrate that tree cover levels above $60 \%$ are possible and urban tree cover between 40 and $60 \%$ is common in forested states. Thus a minimum $40 \%$ urban tree cover goal is attainable in many forest regions. However, at the local scale, as population density and impervious cover increase, lower percent tree cover levels would be expected. Based on the state data, reasonable minimum urban tree cover goals for grassland areas would be $20 \%$ and for deserts, $15 \%$. These goals are near the current average cover percentages and should be attainable by most cities as a minimum. More progressive goals to enhance ecosystem services by trees could be set at higher levels than the minimum and still should be attainable by most cities, but at increased environmental or economic costs depending upon location.

The minimum goals described here are based on the results of this national analysis. However, many local factors determine local tree cover, not least of which is human management desires or goals for an area. Thus local managers should set locally specific tree goals based on local conditions, desires and data sets. The national data sets here could help in providing general guidelines for determining local goals and for comparison of average cover among states. Local tree cover goals and associated management plans can help sustain desired tree cover levels and potentially reverse the recent decline in urban tree cover nationally (Nowak \& Greenfield, 2012).

One way to attain an optimal structure of natural and artificial surfaces in an urban area is to quantify the current conditions and then develop a local management plan to attain the desired structure of trees and impervious surfaces. To help quantify the cover types within an area, a no-cost tool (i-Tree Canopy) is available (www.itreetools.org) that allows users to photo-interpret a city using Google Map ${ }^{\circledR}$ images. This program quantifies the percent cover and associated standard error for each cover class based on interpreter's classification of random points. Photointerpretation is relatively quick, easy and accurate (depending upon the skills of the interpreter), but does not produce a map of the locations of the cover classes, rather just an estimate of the percent or total area occupied by a cover class within a specific geography.

\subsection{Limitations}

Though photo-interpretation provides a relatively simple and inexpensive means to assess tree and impervious cover, there are various limitations to the cover estimates. These limitations generally relate to the ability of the interpreter to accurately distinguish the various cover classes. Differing spatial resolutions and image acquisition dates can potentially affect interpretation, but interpreters could choose to not classify points and $99 \%$ of the points were classified as interpretable. Different interpreters can also potentially classify points differently, but quality control checks were in close agreement. Regardless of whether humans classify images or computers are used to derive cover classes, both methods have limitations. Though photo-interpretation is often more tedious and does not produce a detailed cover map, the human ability to distinguish objects can be used to produce accurate cover estimates in a timely fashion through random sampling. 


\section{Conclusion}

Percent tree and impervious cover differ significantly among states. Urban development increases the amount of impervious cover, but can increase or decrease tree cover depending upon the local environment and human interactions. Urban development in predominantly grassland states tends to increase percent tree cover in contrast to rural lands. Urban development in forested regions tends to decrease percent tree cover relative to rural lands, but the reduction varies among states. These patterns indicate the important role of urban development patterns and social management/planning roles on enhancing or sustaining (reducing the loss of) tree cover in urban areas in the context of the natural environment. Cover data of a city or region can provide a baseline for developing management plans, setting tree cover goals, and for monitoring change through time. As human management is an important factor along with nature in determining the extent of tree cover in urban areas, management plans can be developed to enhance tree cover (and/or reduce impervious cover) and consequently enhance numerous ecosystem services related to human health and well-being. Photo-interpreted data on cover in urban areas and elsewhere can provide an accurate means of assessing cover types and changes in cover through time to help managers and planners make informed decisions on how to better improve local landscapes and the environment.

\section{Acknowledgments}

Funding for this project was provided, in part, by the U.S. Forest Service's RPA Assessment Staff and State \& Private Forestry's Urban and Community Forestry Program. The use of trade, firm, or corporation names in this article is for the information and convenience of the reader. Such use does not constitute an official endorsement or approval by the U.S. Department of Agriculture Forest Service of any product or service to the exclusion of others that may be suitable. We thank Mike Boarman, Chris Sorrentino, Maude Morse, Sebastian Varela, Allison Bodine and Tian Zhou for photo-interpretation. We also thank John Stanovick for his statistical assistance and review, and Anne Cumming and Susan Stein for their review of an early draft of the manuscript.

\section{References}

Anderson, D. G. (1970). Effect of urban development on floods in Northern Virginia. Reston: U.S. Geological Survey. Water Supply Paper No. 2001C

Dwyer, J. F., McPherson, E. G., Schroeder, H. W., \& Rowntree, R. A. (1992). Assessing the benefits and costs of the urban forest. Journal of Arboriculture, 18(5), 227-234.

Dwyer J. F., Nowak D. J., Noble M. H., \& Sisinni, S. M. (2000). Connecting people with ecosystems in the 21st Century: An assessment of our nation's urban forests. U.S. Forest Service General Technical Report PNW-GTR-490, Portland, OR.

Elvidge, C. D., Tuttle, B. T., Sutton, P. C., Baugh, K. E., Howard, A. T., Milesi, C., et al. (2007). Global distribution and density of constructed impervious surfaces. Sensors, 7(9), 1962-1979.

Faulkner, H., Edmonds-Brown, V., \& Green, A. (2000). Problems of quality designation in diffusely polluted urban streams - The case of Pymme's Brook, North London. Environmental Pollution, 109(1), 91-107.
Google Inc. (2011). Google Earth. http://earth.google.com (accessed online May 1, 2007 to March 10, 2010)

Hamilton, G. W., \& Waddington, D. V. (1999). Infiltration rates on residential lawns in Central Pennsylvania. Journal of Soil and Water Conservation, 3rd Quarter. 564-568.

Heisler, G. M., \& Brazel, A. J. (2010). The urban physical environment: temperature and urban heat islands. In J. Aitkenhead-Peterson, \& A. Volder (Eds.), Urban ecosystem ecology (Agronomy monograph)(pp. 29-56). Madison, WI: Soil Science Society of America.

Kuo, F. E., \& Sullivan, W. C. (2001). Environment and crime in the inner city: Does vegetation reduce crime? Environmental Behavior, 33(3), 343-365.

Leopold, D. J. (1968). Hydrology for urban land planning: A guidebook on the hydrologic effects of urban land use. Reston, VA: U.S. Geological Survey. U.S. Geological Survey Circular 554.

Lerner, D. N. (2002). Identifying and quantifying urban recharge: A review. Hydrogeology Journal, 10(1), 143-152.

Lindgren, B. W., \& McElrath, G. W. (1969). Introduction to Probability and Statistics. London: MacMillan.

McHarg, I. L. (1992). Design with nature. New York: John Wiley and Sons, Inc.

McMahon, G., \& Cuffney, T. F. (2000). Quantifying urban intensity in drainage basins for assessing stream ecological conditions. Journal of the American Water Resources Association, 36, 1247-1262.

MRLC. (2010). Multi-resolution land characteristics consortium (MRLC): National land cover database. http://www.mrlc.gov/ (last accessed April 2010)

National Research Council, Committee on Hydrologic Impacts of Forest Management. (2008). Hydrologic effects of a changing forest landscape. Washington, DC The National Academies Press.

Nowak, D. J. (2012). Contrasting natural regeneration and tree planting in fourteen North American cities. Urban Forestry and Urban Greening, http://dx.doi.org/10.1016/j.ufug.2012.02.005

Nowak, D. J., \& Dwyer, J. F. (2007). Understanding the benefits and costs of urban forest ecosystems. In J. Kuser (Ed.), Urban and Community Forestry in the Northeast (pp. 25-46). New York: Springer Science and Business Media.

Nowak, D. J., \& Greenfield, E. J. (2010). Evaluating the National Land Cover Database tree canopy and impervious cover estimates across the conterminous United States: A comparison with photo-interpreted estimates. Environmental Management, 46, 378-390.

Nowak, D. J., \& Greenfield, E. J. (2012). Tree and impervious cover change in U.S. cities. Urban Forestry and Urban Greening, 11, 21-30.

Nowak, D. J., Noble, M. H., Sisinni, S. M., \& Dwyer, J. F. (2001). Assessing the U.S. urban forest resource. Journal of Forestry, 99(3), 37-42.

Nowak, D. J., Rowntree, R. A., McPherson, E. G., Sisinni, S. M., Kerkmann, E., \& Stevens, J. C. (1996). Measuring and analyzing urban tree cover. Landscape and Urban Planning, 36, 49-57.

Oke, T. R. (1989). The micrometeorology of the urban forest. Philosophical Transactions of the Royal Society of London Series B, 324 335-349.

Paul, M. J., \& Meyer, J. L. (2001). Streams in the urban landscape. Annual Review of Ecology and Systematics, 32, 333-365.

Pitt, R., \& Lantri, J. (2000). Infiltration through disturbed urban soils. In W. James (Ed.), Applied modeling of urban water systems (pp. 1-22). Guelph, Ontario: CHI.

Rose, S., \& Peters, N. E. (2001). Effects of urbanization on streamflow in the Atlanta area (Georgia, USA). A comparative hydrological approach. Hydrological Processes, 15, 1441-1457.

Urbonas, B., \& Benik, B. (1995). Stream stability under a changing environment. In E. E. Herricks (Ed.), Stormwater runoff and receiving systems: Impact, monitoring, and assessment (pp. 77-101). New York: CRC Lewis Publishers.

U.S. Census Bureau. (2007). U.S census data. www.census.gov (last accessed: January 2007)

U.S. Environmental Protection Agency. (1983). Results of the Nationwide Urban Runoff Program: Volume 1 - Final Report. Washington, DC: U.S. Environmental Protection Agency, Water Planning Division. NTIS Accession Number: PB84-185552.

Westphal, L. M.(2003). Urban greening and social benefits: A study of empowerment outcomes. Journal of Arboriculture, 29(3), 137-147.

Wolf, K. M. (2003). Public response to the urban forest in inner-city business districts. Journal of Arboriculture, 29(3), 117-126.

Zhu, Z. (1994). Forest density mapping in the lower 48 states: A regression procedure. U.S. Forest Service Research Paper SO-280, New Orleans, LA. 